\title{
Convergence Analysis of Run-to-run Control for a Class of Nonlinear Systems
}

\author{
G. François, B. Srinivasan and D. Bonvin \\ Laboratoire d'Automatique \\ École Polytechnique Fédérale de Lausanne \\ CH-1015 Lausanne, Switzerland.
}

\begin{abstract}
In run-to-run control, measurements from previous runs are used to push the outputs of the current run towards desired set points. From a run-to-run perspective, the classical dynamics get integrated by each run, thereby leading to a static nonlinear input-output map. This paper shows that, when successive linearization of this nonlinear map is used to adapt the run-to-run controller, convergence may not be achieved. However, convergence can be guaranteed if the controller is based on a linear approximation for which the outputs are in-phase (i.e. within $90^{\circ}$ ) with the true outputs. A convergence proof based on Lyapunov approach is provided. The theoretical aspects are illustrated through the simulated meal-to-meal control of blood glucose concentration in diabetic patients.
\end{abstract}

Keywords : Run-to-run control, Sector nonlinearity, Successive linearization, Convergence analysis, Blood glucose control, Diabetes management.

\section{Introduction}

The class of systems where the process is repeated over time has received increasing attention in recent years [3]. Many industrial operations, especially in the areas of batch chemical production, mechanical machining and semiconductor manufacturing, fall under the category of repetitive processes [4, 13].

Run-to-run control is a method that exploits the repetitive nature of processes. The measurements obtained from previous runs are used to adapt the manipulated variables of the current run in order to push the system towards desired set points. These control schemes are very attractive in practice since they only require measurements that are available at the end of the run.

Though dynamics are present within each run, from a runto-run perspective (i.e. upon integration of the within-run dynamics), the map between the manipulated and controlled variables is a static one $[10,6]$. Also, an important feature of run-to-run control schemes is the presence of an implicit one-run delay (also called memory in the literature $[1,11]$ ) between the update of the manipulated variables at the beginning of the run and the measurement of the outputs at the end of the run.

The standard run-to-run control technique uses linearization [7]. The static nonlinear map is linearized at some operating point, for which a linear controller is designed. The difficulty with this technique arises from the fact that the linearization, which is locally valid, may no longer be appropriate when the operating point changes. To handle this difficulty, successive linearization can be used, whereby the linearization is performed around the current operating point. Unfortunately, as will be shown in this paper, this approach does not guarantee convergence. This study considers a class of static nonlinear systems for which linear non-adaptive run-to-run controllers that guarantee convergence can be designed. The class extends the idea of sector nonlinearity that has been widely studied in the literature $[5,12]$. Convergence is proven using a Lyapunov approach.

The paper is organized as follows. In the next section, the run-to-run control of linear and nonlinear systems is briefly reviewed. The class of nonlinearities addressed in this paper and the convergence analysis using Lyapunov approach are provided in Section 3. Section 4 illustrates the theoretical results via an example, and Section 5 concludes the paper.

\section{Run-to-run Control Scheme}

Consider the control of a repetitive dynamic process. The input profiles can be parameterized using a finite (typically low) number of input parameters, $\pi \in \mathfrak{R}^{m}$ [9]. The outputs, $T \in \mathfrak{R}^{p}$, correspond to measurements available at the end of the run. Each run is dynamic. However, since $\pi$ is chosen at the beginning of the run and $T$ is measured at the end of the run, the dynamics get lumped into a static map.

In run-to-run control, the input parameters $\pi$ are modified between consecutive runs so as to eventually meet the set points for the outputs, say $T_{r e f}=0$. A square system is assumed here for simplicity, i.e. $p=m$. It is furthermore assumed that there exists a value $\pi^{*}$ for which $T=T_{r e f}=0$. 
The challenge in run-to-run control arises from the fact that $\pi^{*}$ is typically unknown.

\subsection{Run-to-run Control of Linear Systems}

If the static map is linear, i.e. $T=G\left(\pi-\pi^{*}\right)$, the following result provides an adaptation law that can be used to enforce $\pi_{k}=\pi^{*}$ and $T_{k}=0$ as $k \rightarrow \infty$.

Theorem 1 Let $T=G\left(\pi-\pi^{*}\right)$, where $G$ is full rank. Let the adaptation law be

$$
\pi_{k+1}=\pi_{k}-\gamma G^{-1} T_{k}
$$

where $\gamma$ is a scalar gain, $\pi_{k}$ the input parameters used in the $k^{\text {th }}$ run, and $T_{k}$ the corresponding measurements at the end of the run. Then, for $0<\gamma<2, \pi_{k} \rightarrow \pi^{*}$ and $T_{k} \rightarrow 0$ as $k \rightarrow \infty$.

Proof: Let $\Delta \pi_{k}=\pi_{k}-\pi^{*}$. Then, the update law is given by:

$$
\Delta \pi_{k+1}=\Delta \pi_{k}-\gamma G^{-1} G \Delta \pi_{k}=(1-\gamma) \Delta \pi_{k}
$$

This discrete-time dynamic system is asymptotically stable for $-1<(1-\gamma)<1$, i.e. for $0<\gamma<2$. It follows that $\Delta \pi_{k} \rightarrow 0$ and thus $\pi_{k} \rightarrow \pi^{*}$ and $T_{k} \rightarrow 0$ as $k \rightarrow \infty$.

The general adaptation law is $\pi_{k+1}=\pi_{k}-\Gamma G^{-1} G \pi_{k}$, where $\Gamma$ is an $m \times m$ matrix. However, for simplicity, the special case $\Gamma=\gamma I$, with $\gamma$ a scalar and $I$ the identity matrix, is considered throughout this paper.

\subsection{Run-to-run Control of Nonlinear Systems}

In the case of a nonlinear static map, the successive linearization technique can be used to update the parameters. The gain matrix $G$ is $\left(\frac{\partial T}{\partial \pi}\right)$ computed around the current guess $\pi_{k}$. The adaptation law with successive linearization reads:

$$
\pi_{k+1}=\pi_{k}-\left.\gamma\left(\frac{\partial T}{\partial \pi}\right)\right|_{\pi_{k}} ^{-1} T_{k}
$$

This corresponds to the standard Newton-Raphson algorithm for finding the value of $\pi$ that gives $T=0$. However, this algorithm does not necessarily exhibit global convergence [8]. The update can oscillate around a minimum of $T$, or go towards infinity or some undesired equilibrium points if it is not appropriately bounded.

The absence of global convergence is easy to picture in the scalar case. Suppose that $\left(\frac{\partial T}{\partial \pi}\right)>0$ at the solution $\pi^{*}$ and $T$ has a maximum at $\pi_{1}>\pi^{*}$ as shown in Figure 1. For $\pi<\pi_{1}$, the adaptation law decreases $\pi$, which is in fact the desired update direction. However, for $\pi>\pi_{1}$, the adaptation law would increase $\pi$ in order to reduce $T$. Though this is correct from a local perspective, the actual update is in a direction opposite to the desired one. In other words, global convergence requires more than the local picture provided by the gradient.

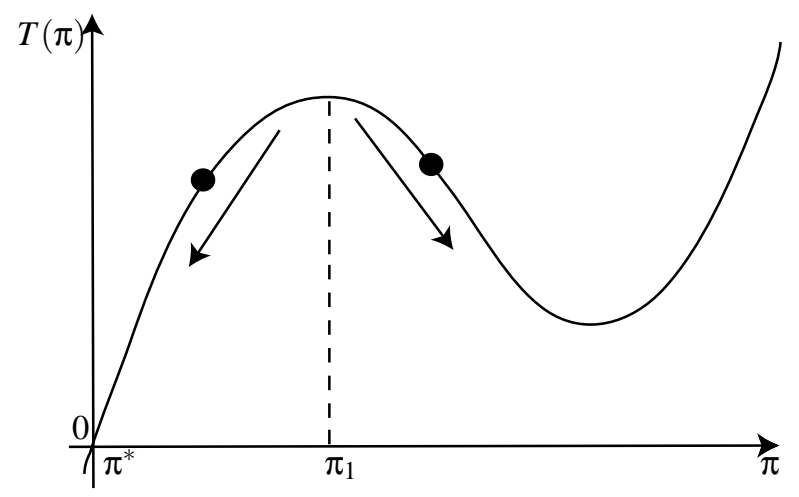

Figure 1: Illustration of possible non-convergence with successive linearization.

\section{Convergence Analysis for a Class of Nonlinear Systems}

A class of nonlinear systems will be defined for which convergence can be achieved with a constant linear adaptation law similar to that for linear systems.

\subsection{Class of Nonlinearities}

This paper considers a class of nonlinearities where there is agreement between the local and global pictures. Note that the linearization is not around the (unknown) solution but rather some arbitrary operating point.

The following assumptions are made:

1. There exists a $\pi^{*}$ for which $T=0$, and

2. There exists a full-rank $m \times m$ matrix $\bar{G}$ and a scalar $\alpha>0$ such that

$$
T^{T} T<\alpha T^{T} \bar{G}\left(\pi-\pi^{*}\right), \quad \forall \pi \neq \pi^{*}
$$

Several remarks about this class of nonlinearities are in order:

- Let $\Delta \pi=\pi-\pi^{*}$. The second assumption implies $T^{T} \bar{G} \Delta \pi>\frac{1}{\alpha} T^{T} T>0$ for all $\Delta \pi \neq 0$. This indicates that the angle between the output vector $T$ and its linear prediction $\bar{G} \Delta \pi$ does not exceed $90^{\circ}$, i.e. the two vectors are said to be in-phase.

- The second assumption also implies unicity of the solution: If $T=0$, for $\Delta \pi \neq 0$, then $\alpha T^{T} \bar{G} \Delta \pi=0$ and $T^{T} T=0$ which violates the strict inequality (4).

- The classical definition of sector nonlinearity is $(T-$ $a \bar{G} \Delta \pi)^{T}(b \bar{G} \Delta \pi-T)>0$, with $0 \leq a \leq b$ [12]. This means that the nonlinearity lies between two linear functions, $a \bar{G} \Delta \pi$ and $b \bar{G} \Delta \pi$, as shown in Figure 2. The condition imposed here is $T^{T} T<\alpha T^{T} \bar{G} \Delta \pi$, which is a limiting case of sector nonlinearity with $a=0$ and $b=\alpha$. 
- A special case of sector nonlinearity is the bounded multiplicative uncertainty, i.e. $T=\bar{G}(1+\Delta G) \Delta \pi$, where $\|\Delta G\|<\beta, \beta>0$. A comparison with the classical definition gives $a=(1-\beta)$ and $b=(1+\beta)$. Due to the imposed symmetry, $a \geq 0$ implies $\beta \leq 1$ and $b \leq 2$.

- A good choice of $\bar{G}$ is the gain matrix at the solution, $G^{*}=\left.\frac{\partial T}{\partial \pi}\right|_{\pi^{*}}$, which unfortunately is usually unknown. However, this choice is not mandatory since it is only necessary to have the two vectors $T$ and $\bar{G}\left(\pi-\pi^{*}\right)$ in-phase. Furthermore, $\alpha$ is chosen so as to appropriately accommodate the nonlinearity.

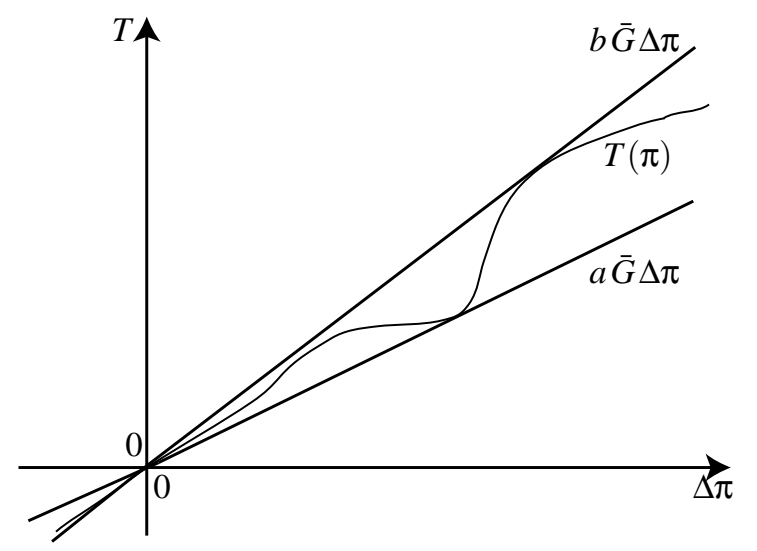

Figure 2: One-dimensional example of sector nonlinearity

\subsection{Convergence Analysis}

Theorem 2 Consider the static map between $\pi$ and $T$ with $T=0$ for $\pi=\pi^{*}$. Let $\bar{G}$ be a full-rank $m \times m$ matrix and $\alpha$ a scalar such that

$$
T^{T} T<\alpha T^{T} \bar{G}\left(\pi-\pi^{*}\right), \quad \forall \pi \neq \pi^{*} .
$$

Also, consider the adaptation law

$$
\pi_{k+1}=\pi_{k}-\gamma \bar{G}^{-1} T_{k}
$$

where $\gamma$ is a scalar gain. For $0<\gamma<\frac{2}{\alpha}, \pi_{k} \rightarrow \pi^{*}$ and $T_{k} \rightarrow 0$ as $k \rightarrow \infty$.

Proof: The proof is based on Lyapunov's direct method [12] and uses the Lyapunov function candidate $V\left(\pi_{k}\right)=$ $\Delta \pi_{k}^{T} \bar{G}^{T} \bar{G} \Delta \pi_{k}$ where $\Delta \pi_{k}=\pi_{k}-\pi^{*}$. The outline of the proof is as follows: First, the fact that $V\left(\pi_{k}\right)>0, \forall \pi_{k} \neq \pi^{*}$ and $V\left(\pi^{*}\right)=0$ is straightforward, since $V$ is quadratic and $\bar{G}$ is full rank.

Next, it will be verified that $V\left(\pi_{k+1}\right)<V\left(\pi_{k}\right), \forall \pi_{k} \neq \pi^{*}$ and $V\left(\pi_{k+1}\right)=V\left(\pi_{k}\right)$ for $\pi_{k}=\pi^{*}$.

For this part, the adaptation law (6) is rewritten as:

$$
\Delta \pi_{k+1}=\Delta \pi_{k}-\gamma \bar{G}^{-1} T_{k}
$$

Consider first the case $\Delta \pi_{k}=0$, i.e. $\pi_{k}=\pi^{*} . T_{k}=0$ by definition of $\pi^{*}$ and $\Delta \pi_{k+1}=\Delta \pi_{k}=0$. Thus, $V_{k+1}=V_{k}=0$.

Consider now the case $\Delta \pi_{k} \neq 0$. Equation (7) can be expressed as:

$$
T_{k}=-\frac{1}{\gamma} \bar{G}\left(\Delta \pi_{k+1}-\Delta \pi_{k}\right)
$$

which, substituted into (4), gives:

$$
\begin{gathered}
\Delta \pi_{k+1}^{T} \bar{G}^{T} \bar{G} \Delta \pi_{k+1}+(\gamma \alpha-2) \Delta \pi_{k}^{T} \bar{G}^{T} \bar{G} \Delta \pi_{k+1} \\
+(1-\gamma \alpha) \Delta \pi_{k}^{T} \bar{G}^{T} \bar{G} \Delta \pi_{k}<0
\end{gathered}
$$

As $\Delta \pi_{k}^{T} \bar{G}^{T} \bar{G} \Delta \pi_{k+1}$ is the scalar product of $\bar{G} \Delta \pi_{k}$ and $\bar{G} \Delta \pi_{k+1}$, the Cauchy-Schwartz inequality can be used [8]:

$$
\Delta \pi_{k+1}^{T} \bar{G}^{T} \bar{G} \Delta \pi_{k+1} \geq \frac{\left(\Delta \pi_{k}^{T} \bar{G}^{T} \bar{G} \Delta \pi_{k+1}\right)^{2}}{\Delta \pi_{k}^{T} \bar{G}^{T} \bar{G} \Delta \pi_{k}}
$$

Substituting (10) into (9) gives:

$$
\begin{array}{r}
\left(\Delta \pi_{k}^{T} \bar{G}^{T} \bar{G} \Delta \pi_{k+1}+(\gamma \alpha-1) \Delta \pi_{k}^{T} \bar{G}^{T} \bar{G} \Delta \pi_{k}\right) \\
\left(\Delta \pi_{k}^{T} \bar{G}^{T} \bar{G} \Delta \pi_{k+1}-\Delta \pi_{k}^{T} \bar{G}^{T} \bar{G} \Delta \pi_{k}\right)<0
\end{array}
$$

Since the difference between the two terms in (11) is :

$$
\gamma \alpha \Delta \pi_{k}^{T} \bar{G}^{T} \bar{G} \Delta \pi_{k}>0
$$

the first term of (11) has to be positive and the second term negative for the inequality to be verified. So,

$$
\Delta \pi_{k}^{T} \bar{G}^{T} \bar{G} \Delta \pi_{k+1}>(1-\gamma \alpha) \Delta \pi_{k}^{T} \bar{G}^{T} \bar{G} \Delta \pi_{k}
$$

and :

$$
\Delta \pi_{k}^{T} \bar{G}^{T} \bar{G} \Delta \pi_{k+1}<\Delta \pi_{k}^{T} \bar{G}^{T} \bar{G} \Delta \pi_{k}
$$

For $0<\gamma<\frac{2}{\alpha}$, it follows that :

$$
-1<(1-\gamma \alpha)<\frac{\Delta \pi_{k}^{T} \bar{G}^{T} \bar{G} \Delta \pi_{k+1}}{\Delta \pi_{k}^{T} \bar{G}^{T} \bar{G} \Delta \pi_{k}}<1
$$

Noting that $(2-\gamma \alpha)>0$, and substituting $\Delta \pi_{k}^{T} \bar{G}^{T} \bar{G} \Delta \pi_{k+1}<$ $\Delta \pi_{k}^{T} \bar{G}^{T} \bar{G} \Delta \pi_{k}$ into (9) gives:

$$
\begin{aligned}
& \Delta \pi_{k+1}^{T} \bar{G}^{T} \bar{G} \Delta \pi_{k+1}<(\gamma \alpha-1) \Delta \pi_{k}^{T} \bar{G}^{T} \bar{G} \Delta \pi_{k} \\
& \quad+(2-\gamma \alpha) \Delta \pi_{k}^{T} \bar{G}^{T} \bar{G} \Delta \pi_{k+1}<\Delta \pi_{k}^{T} \bar{G}^{T} \bar{G} \Delta \pi_{k}
\end{aligned}
$$

Thus, it has been shown that $V_{k+1}<V_{k}$, for all $\Delta \pi_{k} \neq 0$.

Additional remarks can be made:

- The condition that guarantees convergence of successive linearization is of local nature, i.e. the elements 
of $\left.\frac{\partial T}{\partial \pi}\right|_{\pi_{k}}$ and the corresponding elements of $\left.\frac{\partial T}{\partial \pi}\right|_{\pi^{*}}$ must have the same sign. In contrast, the proposed method relies on the global condition (4) that can be satisfied even if some elements of $\left.\frac{\partial T}{\partial \pi}\right|_{\pi_{k}}$ and the corresponding elements of $\bar{G}$ have different signs.

- A larger value of $\alpha$ calls for a smaller $\gamma$, and thus slower adaptation. Hence, $\alpha$ should be chosen as the smallest value that satisfies (4).

\section{Illustrative Example}

The meal-to-meal control of blood glucose concentration through insulin injection in a diabetic patient is considered. The simple dynamic model presented in [2] is used. This model correctly captures the essential features: After a meal, the glucose concentration in blood increases up to a certain level before decreasing. Also, there could be some undershoot before the glucose concentration settles to its final value.

The objective of meal-to-meal control is to adapt both the concentration of the injected insulin and the injection time so as to achieve preset maximum and minimum glucose concentrations [6]. Every meal cycle is considered as a run. Measurements from the previous meal cycle are used to adjust the current cycle.

\subsection{Model of the System}

The model is based on the assumption that there is a 3rdorder linear dynamic relationship between the concentration of the injected insulin and the insulin concentration in blood. Also, the meal has a 2nd-order effect on the blood glucose concentration. The model equations read:

$$
\begin{array}{rlrl}
\dot{x}_{1} & =p_{7} x_{5}-p_{1} x_{1}-p_{6} x_{2}\left(x_{1}+G_{b}\right), & & x_{1}(0)=0 \\
\dot{x}_{2} & =p_{2}\left(x_{3}-x_{2}\right), & & x_{2}(0)=0 \\
\dot{x}_{3} & =p_{3}\left(x_{4}-x_{3}\right), & x_{3}(0)=0 \\
\dot{x}_{4} & =p_{4}\left(I(t)-x_{4}\right), & & x_{4}(0)=0 \\
\dot{x}_{5} & =p_{5}\left(M(t)-x_{5}\right), & & x_{5}(0)=0
\end{array}
$$

where $x_{1}$ is the deviation from the standard blood glucose concentration $G_{b}, x_{2}$ to $x_{4}$ filtered concentrations of insulin in blood, and $x_{5}$ the calories accumulated during the meal. $I(t)$ is the insulin concentration profile to be adjusted, and $M(t)$ the calorific contribution of the meal. The variables $x_{1}$ to $x_{4}, G_{b}$ and $I(t)$ are expressed in [mg/dl], while $x_{5}$ and $M(t)$ are expressed in [cal]. The numerical values of the model parameters $p_{1}$ to $p_{7}$ are given in Table 1 .

Since the model parameters vary from person to person, the idea of run-to-run control is to use the measurements from the previous meal cycle to iteratively determine the insulin injection profile without knowledge of the patient parameters.
Table 1: Model parameters

\begin{tabular}{c|c}
\hline parameter & value \\
\hline$p_{1}$ & $\frac{1}{100} \mathrm{~min}^{-1}$ \\
$p_{2}$ & $\frac{1}{30} \mathrm{~min}^{-1}$ \\
$p_{3}$ & $\frac{1}{7} \mathrm{~min}^{-1}$ \\
$p_{4}$ & $\frac{1}{60} \mathrm{~min}^{-1}$ \\
$p_{5}$ & $\frac{1}{80} \mathrm{~min}^{-1}$ \\
$p_{6}$ & $6.68 \times 10^{-7} \mathrm{dl} /(\mathrm{mg} \cdot \mathrm{min})$ \\
$p_{7}$ & $0.012 \mathrm{mg} /(\mathrm{dl} . c a l . m i n)$ \\
$G_{b}$ & $80 \mathrm{mg} / \mathrm{dl}$ \\
\hline
\end{tabular}

\subsection{Input Parameterization and Meal Model}

As shown in Figure 3, the input $I(t)$ can be parameterized using the two parameters, $I_{\text {amp }}$ that represents the amplitude of insulin injection and $t_{i n j}$ the injection time. The duration of the injection is fixed at $1 \mathrm{~min}$. The meal profile $M(t)$ is modeled in a trapezoidal manner, as shown in Figure 4 and with the parameter values provided in Table 2. The final time $t_{f}$ is supposed fixed, and no measurements are available during the cycle.

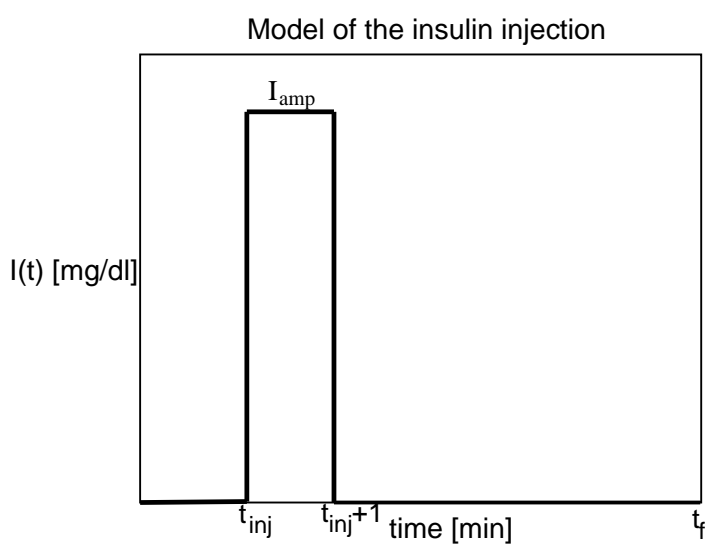

Figure 3: Insulin concentration profile

Table 2: Parameters of the meal profile

\begin{tabular}{c|c}
\hline parameter & value \\
\hline$M_{a m p}$ & $360 \mathrm{cal}$ \\
$t_{1}$ & $5 \mathrm{~min}$ \\
$t_{2}$ & $35 \mathrm{~min}$ \\
$t_{3}$ & $145 \mathrm{~min}$ \\
$t_{4}$ & $175 \mathrm{~min}$ \\
$t_{f}$ & $1000 \mathrm{~min}$ \\
\hline
\end{tabular}

\subsection{Control Objectives and Manipulated Variables}

A typical evolution of the glucose concentration in blood is shown in Figure 5. 


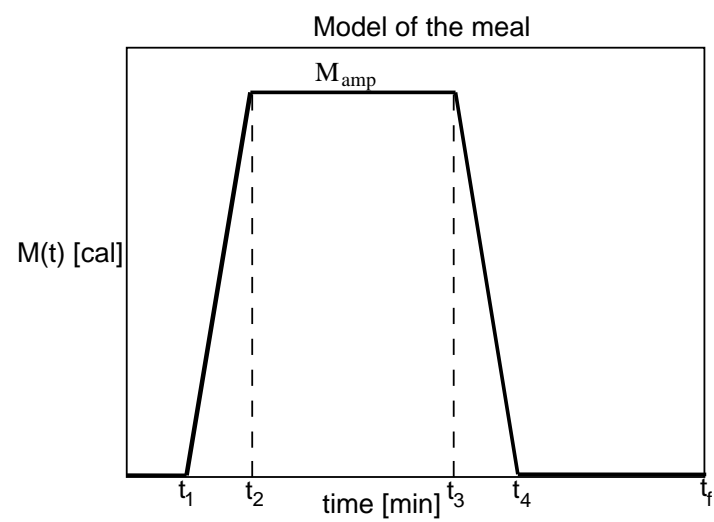

Figure 4: Calorific contribution profile of a meal

The two controlled variables are:

$$
\begin{aligned}
T_{1}: G_{\text {min }} & =\min _{t>t_{\text {inj }}}\left(x_{1}(t)\right) \\
T_{2}: G_{\text {max }} & =\max _{t}\left(x_{1}(t)\right)
\end{aligned}
$$

The values of $T_{1}$ and $T_{2}$, are assumed to be available at the end of each meal cycle. The corresponding set points are: $T_{r e f, 1}=-3.5 \mathrm{mg} / \mathrm{dl}$ and $T_{r e f, 2}=75 \mathrm{mg} / \mathrm{dl}$. The two manipulated variables are $\pi_{1}=I_{a m p}$ and $\pi_{2}=t_{i n j}$.

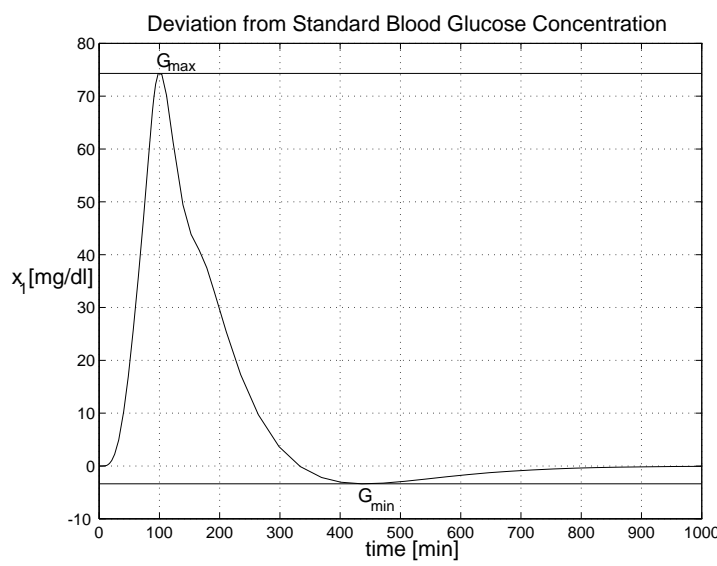

Figure 5: Evolution of glucose concentration within a meal cycle.

\subsection{Results and Discussion}

The results with (i) the successive linearization approach, and (ii) the proposed approach with a single linear controller are compared next. Adaptations starting from two different initial guesses are presented: (a) $I G_{a}: I_{a m p}=$ $3.5 \times 10^{6} \mathrm{mg} / \mathrm{dl}$ and $t_{i n j}=75 \mathrm{~min}$, and (b) $I G_{b}: I_{a m p}=$ $5.05 \times 10^{6} \mathrm{mg} / \mathrm{dl}$ and $t_{i n j}=50 \mathrm{~min}$. For the first guess, both approaches converge to the desired set points, while with the second guess only the proposed approach converges. In all simulations, a 5\% zero-mean gaussian measurement noise on $G_{\min }$ and $G_{\max }$ is assumed.

The results with the initial guess $I G_{a}$ are presented in Figures 6 and 7. It can be seen that both approaches converge to the desired set points, the oscillations around the set points being due to noise. Since the oscillation amplitudes can be reduced by lowering the adaptation gain, $\gamma=0.3$ is used for the five first runs and $\gamma=0.075$ thereafter to avoid noise amplification.

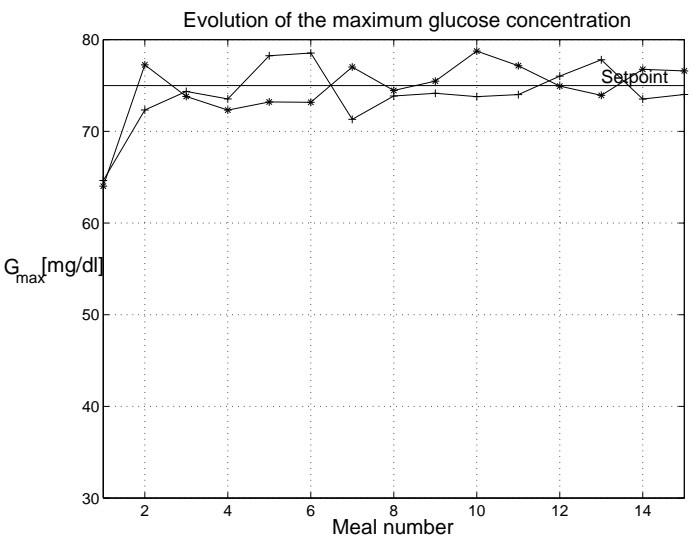

Figure 6: Evolution of the maximum glucose concentration $I G_{a},(+)$ successive linearization, $(*)$ single linear controller

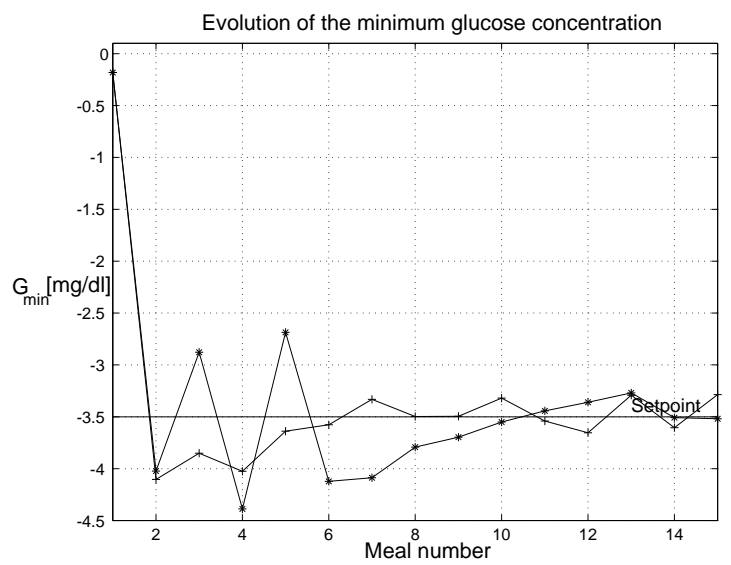

Figure 7: Evolution of the minimum glucose concentration $-I G_{a}$, $(+)$ successive linearization, $(*)$ single linear controller

A key element of the proposed approach is the choice of $\bar{G}$. Here, $\bar{G}$ is computed as the linearization around a point close to the solution, $I_{a m p}=4.7 \times 10^{6} \mathrm{mg} / \mathrm{dl}, t_{i n j}=80 \mathrm{~min}$, which gives:

$$
\bar{G}=\left[\begin{array}{cc}
-3.48 \times 10^{-6} & -1.82 \times 10^{-1} \\
-1.40 \times 10^{-6} & 1.33
\end{array}\right]
$$

It was also verified that $\bar{G}$ satisfies (4) with $\alpha=2$.

The solution computed a posteriori, $I_{\text {amp }}^{*}=4.582 \times$ $10^{6} \mathrm{mg} / \mathrm{dl}, t_{i n j}^{*}=83.94 \mathrm{~min}$, gives the linear map :

$$
G^{*}=\left[\begin{array}{cc}
-3.56 \times 10^{-6} & -2.47 \times 10^{-1} \\
-1.46 \times 10^{-6} & 1.269
\end{array}\right]
$$

It turns out that the successive linearization approach works well since the linearization around the initial guess $I G_{a}$ has elements with the same signs as the corresponding elements 
in $G^{*}$ :

$$
\bar{G}_{I G_{a}}=\left[\begin{array}{cc}
-8.54 \times 10^{-7} & -3.60 \times 10^{-2} \\
-3.17 \times 10^{-6} & 1.28
\end{array}\right]
$$

The situation is different when starting from $I G_{b}$, for which the results are presented in Figures 8 and 9. The proposed approach converges to the desired set points, while successive linearization does not. This is due to the fact that, in the linearization around $I G_{b}$, one of the elements has a sign opposite to that of the corresponding element in $G^{*}$ :

$$
\bar{G}_{I G_{b}}=\left[\begin{array}{cc}
-7.78 \times 10^{-6} & -6.03 \times 10^{-2} \\
-1.33 \times 10^{-6} & -5.05 \times 10^{-1}
\end{array}\right]
$$

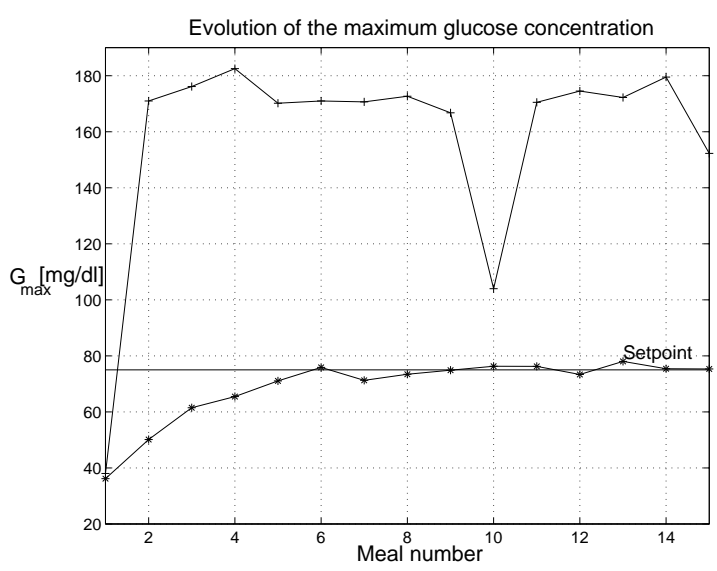

Figure 8: Evolution of the maximum glucose concentration $I G_{b},(+)$ successive linearization, $(*)$ single linear controller

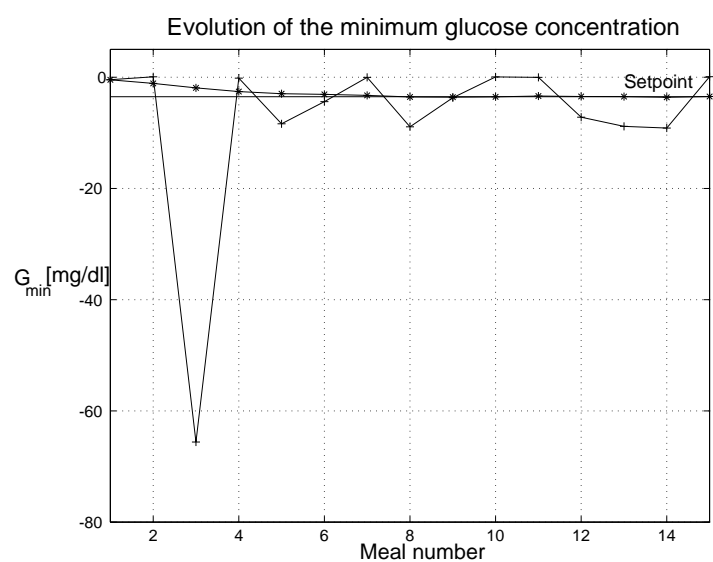

Figure 9: Evolution of the minimum glucose concentration - $I G_{b}$, $(+)$ successive linearization, $\left.{ }^{*}\right)$ single linear controller

Note that, when the methods converge, convergence is fairly fast (within 6 meals) despite the presence of noise.

\section{Conclusions}

This work has demonstrated the effectiveness of run-to-run control applied to a class of nonlinear systems. Conditions have been provided that guarantee convergence using a single linear controller. The basic assumption is that the linear approximation used to predict the outputs be in-phase with the true outputs. The convergence results were illustrated through the meal-to-meal control of blood glucose concentration in diabetic patients.

Since the successive linearization approach, which looks intuitively appealing, does not converge from all initial conditions, future research could investigate conditions that guarantee its convergence. Also, convergence analysis for other classes of nonlinear systems is envisaged.

\section{References}

[1] S. Arimoto and T. Naniwa. Learnability and adaptability from the viewpoint of passivity analysis. Intelligent Automation and Soft Computing, 8(2):71-94, 2002.

[2] R.N. Bergman, L.S. Phillips, and C. Cobelli. Physiologic evaluation of factors controlling glucose tolerance in man. J. Clin. Invest.,, 68:1456-1467, 1981.

[3] W.J. Campbell, S.K. Firth, A.J. Toprac, and T.F. Edgar. A comparison of run-to-run algorithms. In American Control Conference, pages 4212-4217, Anchorage, 2002.

[4] E.D. Castillo and A.M. Hurwitz. Run-to-run process control: Literature review and extensions. Journal of Quality Technology, 29:184-196, 1997.

[5] T. Chu, L. Huang, and L. Wang. Guaranteed absolute stability of a class of delay systems with local sector nonlinearities via piecewise linear Lyapunov function. In American Control Conference, pages 4212-4217, Arlington, 2001.

[6] F. Doyle III, B. Srinivasan, and D. Bonvin. Run-torun control strategy for diabetes management. In IEEE Engineering in Medecine and Biology Conference, Istanbul, 2001.

[7] G. Francois, B. Srinivasan, and D. Bonvin. Run-torun optimization of an emulsion polymerization reactor. In IFAC, page 1258, Barcelona, Spain, 2002.

[8] E. Kreysig. Advanced Engineering Mathematics, Sixth Edition. John Wiley And Sons, Inc, New York, 1988.

[9] B. Srinivasan, S. Palanki, and D. Bonvin. Dynamic optimization of batch processes: I. Characterization of the nominal solution. Comp. Chem. Eng., 27:1-26, 2003.

[10] B. Srinivasan, C. J Primus, D. Bonvin, and N. L. Ricker. Run-to-run optimization via generalized constraint control. Control Eng. Practice, 9:911-919, 2001.

[11] A. Tayebi and M.B. Zaremba. Robust iterative learning control design is straightforward for uncertain lit systems satisfying the robust performance condition. IEEE Trans. Automat. Contr., 48(1):101-106, 2003.

[12] M. Vidyasagar. Nonlinear Systems Analysis, Second Edition. Prentice Hall, New Jersey, 1993.

[13] E. Zafiriou, R.A. Adomaitis, and G. Gattu. Approach to run-to-run control for rapid thermal processing. In American Control Conference, pages 1286-1288, Seattle, 1995. 